\title{
In Vitro Quantification of the Radiopacity of Onyx during Embolization
}

\author{
Yuan Yuan Jiang, MD', Ye-eun Jo, BS², Jung Min Woo, BS², Ok Kyun Lim, BS², \\ Changmo Hwang, PhD's, Jun Young Maeng, BS ${ }^{2}$, Jieun Kim, MS ${ }^{2,4}$, \\ Namkug Kim, PhD ${ }^{2,4}$, Deok Hee Lee, MD $^{2}$
}

Purpose: Onyx has been successfully applied in the treatment of various neurovascular lesions. However, some experience is required to get accustomed to its unpredictable fluoroscopic visibility during injection. This in vitro study aimed to evaluate the characteristics of radiopacity change in a simulated embolization procedure.

Materials and Methods: Using a bench-top Onyx injection experiment simulating a typical brain arteriovenous malformation embolization, nine cycles of casting modes (continuous injection) and plugging modes (injection with intermittent pauses) were performed. Radiodensity of Onyx droplets collected from the microcatheter tip and the distal head portion of the microcatheter were measured as time lapsed. Distribution of droplet radiodensity (radiodensity) and distribution of radiographic grade (grade) were analyzed and compared by repeated measurements.

Results: Within-group analysis revealed no significant radiodensity change with time $(P>0.05)$. The radiodensity was significantly higher in the casting mode than in the plugging mode $(P<0.01)$. The lateral radiograph of the microcatheter showed higher radiopacity $(P<0.01)$ and better evenness $(P<0.01)$ in the casting mode than in the plugging mode. A significant difference in microcatheter attenuation (both radiographic grade mean and $S D ; P<0.01$ ) was noted between the two modes. Radiodensity had a significant influence on the radiopacity and radiopacity evenness of the microcatheter.

Conclusion: The radiopacity of the Onyx can vary significantly over time because of early precipitation of tantalum powder. Radiopacity decreased significantly during plugging modes, characterized by pauses between injections.

Key Words : Onyx; Liquid embolic material; Brain arteriovenous malformations; Embolization

'Department of Medical Biotechnology, Dongguk University, Ilsan, Korea

2Department of Radiology, Research Institute of Radiology, Asan Medical Center, University of Ulsan College of Medicine, Seoul, Korea

${ }^{3}$ Biomedical R\&D Center, Asan Institute for Life Sciences, Asan Medical Center, Seoul, Korea

${ }^{4}$ Department of Convergence Medicine, Asan Medical Center, University of Ulsan College of Medicine, Seoul, Korea

Received September 24, 2016; accepted after revision January 23, 2017.

Correspondence to: Deok Hee Lee, MD, PhD, Department of Radiology, Asan Medical Center, University of Ulsan College of Medicine, 88 Olympic-ro 43-gil, Songpa-gu, Seoul 05505, Korea.

Tel. 82.2.3010.5944 Fax. 82.2.476.0090 E-mail: dhlee@amc.seoul.kr

This is an Open Access article distributed under the terms of the Creative Commons Attribution Non-Commercial License (http://creativecommons.org/licenses/by-nc/3.0) which permits unrestricted non-commercial use, distribution, and reproduction in any medium, provided the original work is properly cited. 
Onyx ${ }^{\mathrm{TM}}$ (Covidien, Irvine, CA, USA), a liquid embolic material, has been used successfully for endovascular treatment of various neurovascular lesions, such as brain arteriovenous malformation and dural arteriovenous fistula [1-3]. Different from glue (Histoacryl, B. Braun, Melsungen, Germany), an adhesive embolic material, Onyx provides considerable procedural versatility owing to its non-adherent nature. It was first introduced by Yamashita et al. and Terada et al. [4, 5] and is currently one of the commonly used liquid embolic agents for neurointervention. It is a mixture of ethylene and vinyl alcohol (EVOH copolymer) dissolved in dimethyl sulfoxide (DMSO) at three different concentrations, each resulting in a different viscosity of the mixture at 18,20, and 34 centistokes, respectively. Micronized tantalum powder (35\% weight/ volume) is added to make the highly radiolucent mixture radiopaque.

Owing to the excellent radiopacity of the tantalum powder, well-shaken (after 20 min of agitation) Onyx exhibits very good radiopacity. However, due to the significant weight difference between the metallic powder and the liquid part, the mixture tends to separate easily mostly due to early sedimentation of the metallic component, making it difficult to keep the mixture homogeneous during embolization [6]. The advantages of Onyx are offset by poor radiopacity during injection. We have experienced significantly low radiopacity (and often followed by very high radiopacity) of Onyx, especially during slow injection and while resuming injection after an injection pause, both of which are steps usually required for the "plug and push" technique [6].

Early sedimentation of the tantalum powder within the microcatheter lumen and injecting syringe probably causes frequent radiopacity inhomogeneity of wellshaken Onyx. It is easy to note alternating low- and high-radiopacity segments of the injecting microcatheter on the fluoroscopy, especially in the dependent segments of the multiple-curved microcatheter. As a result of poor radiopacity, a significant reflux of less opaque Onyx might cause difficulty in microcatheter removal or a disastrous parent artery occlusion [7].

We hypothesized that the inhomogeneity of Onyx radiopacity, which is currently inevitable for achieving optimal occlusion of the target lesion, was exaggerated when the injection was slow and/or stagnant. This in vitro experiment aimed to observe radiodensity (X-ray attenuation value) changes of Onyx during injection and pause periods using a simplified simulation procedure of a brain arteriovenous malformations embolization to better understanding of the behavior of the mixture in a real situation.

\section{MATERIALS AND METHODS}

\section{In vitro bench-top experiment setting}

The bench-top in vitro experiment was designed to simulate the real process during a typical arteriovenous malformation embolization using Onyx. A radiolucent plate was printed with a real-scale CT image of a midline sagittal head and thorax (Fig. 1A), and placed on the angiography machine (Axiom Artis biplane; Siemens, Erlangen, Germany) table simulating the position of a patient during embolization. A microcatheter (Apollo; Covidien, Irvine, CA, USA) was adhered to the plate along the imaginary course of the aortoiliac artery path all the way up to the main anterior cerebral artery feeder of a medial parietal cortical arteriovenous malformation. The lower ("thigh") portion was a clamp stand simulating femoral puncture and preventing any motion of the microcatheter. At the distal ("head") portion, an approximately $5 \mathrm{~cm}$ microcatheter was extended out of the plate margin. A 48-well plate (SPL Life Science, Gyeonggi-do, Korea) pre-filled with $0.3 \mathrm{~mL}$ of distilled water was placed below the tip to collect the Onyx droplets. The buoyancy of the water kept the Onyx droplets in a round shape (Fig. 1B-D).

\section{Onyx preparation, injection, and collection}

Onyx 18 was injected using $1.0 \mathrm{~mL}$ syringes after 20 min of maximum agitation. Before injection, the microcatheter lumen was rinsed with dimethyl sulfoxide (DMSO) sufficiently and then connected with a 1 $\mathrm{mL}$ syringe full of well-agitated Onyx. To simulate the injection conditions of a typical arteriovenous malformation embolization procedure, we set two different injection modes in one cycle - a casting mode and a plugging mode. Nine cycles of casting mode plus plugging mode with the same experiment settings were performed.

In both modes, the Onyx was injected continuously at a rate of $0.05 \mathrm{~mL} / \mathrm{min}$. A single cycle started with a casting mode, which continued until 35 droplets were collected, simulating the period when Onyx is injected continuously into the nidus. Then, the plugging mode started. It simulated a period when reflux of the Onyx was found. A plug should form for stable injection, and multiple, brisk injection-and-waiting steps were necessary to build up a solid plug around the tip of the microcatheter. Therefore, five repetitions of a 1-min 
pause followed by a 2-second (s) injection were done, resulting in five droplets in one plugging mode. In other words, right after collecting the 35th droplet of Onyx in casting mode, Onyx injection was halted for 1 min followed by a brisk injection for $2 \mathrm{~s}$.

To minimize sedimentation of heavy tantalum powder in the injecting syringe, an infusion pump (NE1000 Programmable Syringe Pump; Parkland Scientific, Coral Springs, FL, USA) loaded with the injecting syringe was continuously flipped over manually during injection. A total of $3 \mathrm{~mL}$ of Onyx was infused without significant interruption by prompt switching of the syringes as rapidly as possible for continuous injection.

\section{Radiographic analysis}

The head portion of the microcatheter was radiographed using the lateral $\mathrm{C}$-arm at multiple time points during Onyx injection. Radiographic acquisition was automatically set by the automatic exposure control of the angiography machine at 70.0 kilovolt
$(\mathrm{kV}), 31.0$ milliamps $(\mathrm{mA})$, and 3.5 millisecond (ms) using a small focal spot without copper filtering. Radiographs were taken at every 5 droplets during the casting mode and at every droplet during the plugging mode, resulting in seven images in casting mode and five in plugging mode in every cycle. Injection duration was obtained from the radiographic exposure time in radiograph data.

The head portion of the microcatheter on the radiograph was arbitrarily divided into 12 segments for semi-quantitative analysis of the radiopacity of the running Onyx in the lumen of the head portion of the microcatheter. An experienced radiologist graded the radiopacity of each segment according to the grayness level. Grade 0 meant no significant or low radiopacity; grade 1, less than half; and grade 2, more than half of the length of the segment showed high radiopacity (Fig. 2).

All the collected droplets from 15-well plates were radiographed under the same radiographic conditions listed. The X-ray attenuation of each droplet (droplet radiodensity) was measured by drawing a region of
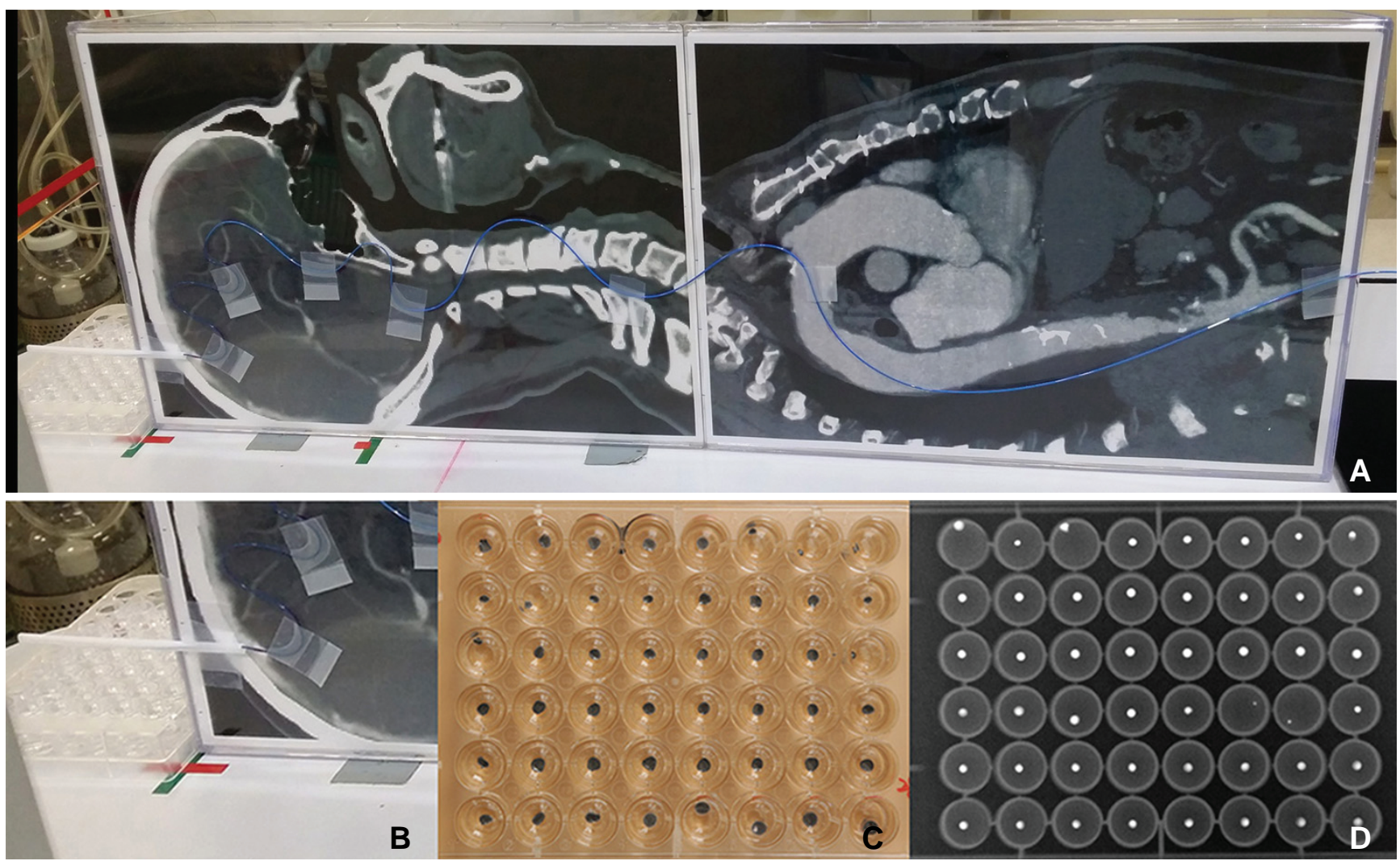

Fig. 1. (A) The bench-top set up of the in vitro experiment simulating the head and body of a patient during a typical brain AVM embolization using Onyx. The microcatheter was adhered to the plate along the imaginary course of the main feeder of a medial parietal cortical brain AVM. Under the very tip of the injecting microcatheter a 48-well-cell-culture plate was placed to collect the Onyx droplets to be radiographed. (B, C, D) Collection of the Onyx droplets (B) Close-up image showing the 48-well-cell-culture plate placed under the injecting microcatheter tip. (C) Image of a 48-well-cell-culture plate filled with Onyx droplets in each well. (D) The radiograph of the same culture plate filled with Onyx droplets. This image was transferred to ImageJ for the measurement of the radiodensity of each droplet. 
interest (ROI) using Image $\mathrm{J}(\mathrm{NIH}$; Bethesda, Maryland; http://imagej.nih.gov/ij/) by an experienced radiology technician. Image magnification was allowed for the accurate demarcation of the droplet margin. A circular margin was used to fit the droplets in most cases; however, rough adjustment was allowed when the margin was not circular to fit.

\section{Data Analysis and statistics}

\section{Onyx droplet radiodensity (droplet radiodensity)}

With the measured radiodensity of the collected Onyx droplets (droplet radiodensity), we drew a timeradiodensity curve based on the time lapsed (the time interval between each drop collection point in a cycle and the starting point of the cycle). Repeated measures analyses (SPSS version 20, Armonk, New York, USA/ Stata 12.0 for Mac (64-bit Intel), StataCorp LP, College Station, TX, USA) were performed to determine the difference of radiodensities within each mode of one cycle (within-group) and the difference between the two modes in one cycle (between-group).

\section{Microcatheter radiographic grade}

Semi-quantitative analysis was performed comparing serial lateral radiographic images of the head portion of the microcatheter and the dynamic change of the X-ray attenuation within the microcatheter lumen. The grade mean (mean) and grade standard deviation (SD) for each radiograph of the microcatheter were calculated with the mean representing the total visualization of the head portion of the microcatheter, and the SD representing the evenness of radiopacity in the segment.

The distributions of mean and SD were drawn according to a time point. Repeated measures were performed to show the difference of mean or SD within each mode of one cycle (within-group), and also the difference between the two modes in one cycle (between-group).

\section{Association between radiodensity and grades}

A scatter plot was drawn to show the association between groups of radiodensities and their corresponding grades (mean or SD) for both casting mode and plugging mode. Pearson's Correlation analysis was carried out for both modes.

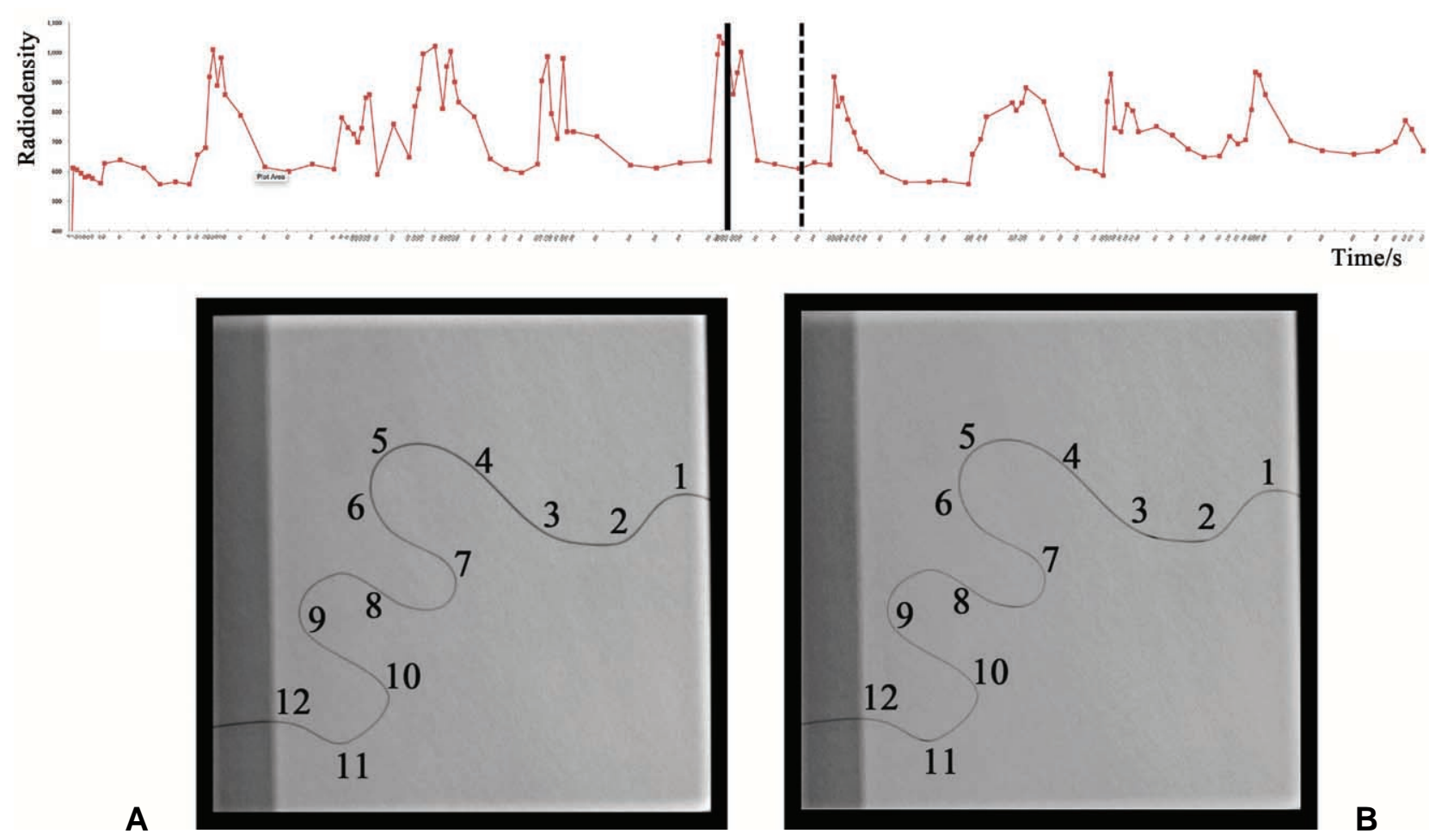

Fig. 2. Time-radiodensity curves of all nine Onyx injection cycles (upper row) and representative radiographic images of the distal head portion of the microcatheter in (A) casting mode (black solid line) and (B) plugging mode (black dash line) of the 5th cycle. An X-ray attenuation (radiodensity) curve for each droplet from all casting and plugging mode cycles demonstrating the dynamic change of $X$-ray units with time lapse and dramatic change of the radiodensity in the two modes. We graded all 12 segments of image $A$ as grade 2 . Image B segments were grade 0 , except for segments 3,8 , and 11, which were grade 1. 


\section{RESULTS}

\section{Droplet radiodensity}

Fig. 3A shows the changes in radiodensities over time in both modes by repeated measurements. The results of radiodensity distribution and the statistical analysis are summarized in Table 1. No significant difference in radiodensity distribution was found in either mode; however, a significant difference $(\mathrm{P}<0.01)$ in radiodensity was present between the two modes.

\section{Microcatheter radiographic grade}

Fig. 2 shows the difference between a lateral radiograph in a casting mode and plugging mode together with the corresponding droplet radiodensity marked on the radiodensity curve in the whole cycle of experiment. In the casting mode, the microcatheter was filled with much more tantalum than in the plugging mode and the lumen appeared to be more even. In the plugging mode, the radiodensity was apparently reduced more than in the casting mode. Moreover, the radiodensities of the curves varied from each other; the lower curves accumulated more tantalum than the higher ones and ended with more attenuation. The mean and SD distribution and the statistical analysis are summarized in Table 1. Fig. 3B and $\mathrm{C}$ show the change of mean and SD in both modes by repeated measurements, respectively. Repeated measurements revealed no significant distribution trend of mean with time for both modes. There was no significant distribution trend of SD with time in the casting mode, but a significant reduction of SD with time in the plugging mode $(\mathrm{P}<0.01)$ was seen. In addition, a significant difference was shown between the two modes in both mean and $\mathrm{SD}(\mathrm{P}<0.01)$.

\section{Relationship between radiodensity and grade}

In the casting mode, as radiodensities increased, the means appeared to increase (Fig. 3D) while the SDs

Table 1. Summary of Statistical Analysis Droplet Radiodensity and Microcatheter Radiographic Distribution

\begin{tabular}{|c|c|c|c|}
\hline \multirow[t]{2}{*}{ Section } & \multirow[t]{2}{*}{ Statistical Analysis Method } & \multicolumn{2}{|l|}{ Group } \\
\hline & & Casting & Plugging \\
\hline \multirow[t]{2}{*}{ Droplet radiodensity } & Within-group Difference* & $\begin{array}{l}\text { Descriptive All radiodensities ranged } \\
\text { between } 600 \text { and } 1100 \mathrm{XU}^{\star *} \text {; no trend } \\
\text { distribution observed with time. } \\
\text { Statistical } \mathrm{P}>0.05\end{array}$ & $\begin{array}{l}\text { Descriptive All radiodensities ranged } \\
\text { between } 500 \text { and } 900 \mathrm{XU} \text {; no trend } \\
\text { distribution observed with time. } \\
\text { Statistical } \mathrm{P}>0.05\end{array}$ \\
\hline & Between-group Difference ${ }^{\dagger}$ & $P<0.01$ & \\
\hline \multirow[t]{4}{*}{$\begin{array}{l}\text { Microcatheter } \\
\text { radiographic grade }\end{array}$} & \multirow{2}{*}{ Within-group Difference ${ }^{\S}$} & $\begin{array}{l}\text { Descriptive All means ranged between } \\
0 \text { and } 25 ; \text { no trend distribution } \\
\text { observed with time. } \\
\text { Statistical mea }{ }^{\dagger+}: P>0.05\end{array}$ & $\begin{array}{l}\text { Descriptive All means ranged between } \\
2 \text { and } 14 \text {; no trend distribution } \\
\text { observed with time. } \\
\text { Statistical mean: } P>0.05\end{array}$ \\
\hline & & $\begin{array}{l}\text { Descriptive All SDs ranged between } \\
0.4 \text { and } 0.6 \text {; no trend distribution } \\
\text { observed with time. } \\
\text { Statistical SD": P>0.05 }\end{array}$ & $\begin{array}{l}\text { Descriptive All SDs ranged between } \\
0.4 \text { and } 0.6 \text {; no trend distribution } \\
\text { observed with time. } \\
\text { Statistical SD: } P<0.01\end{array}$ \\
\hline & \multirow{2}{*}{ Between-group Difference" } & mean: $P<0.01$ & \\
\hline & & SD: $P<0.01$ & \\
\hline \multirow{2}{*}{$\begin{array}{l}\text { Correlation: lateral } \\
\text { radiograph grade and } \\
\text { droplet radiodensity }\end{array}$} & Grade mean \& radiodensity & Correlation coefficient: $0.435, P<0.01$ & Correlation coefficient: $0.295, P<0.05$ \\
\hline & Grade SD \& radiodensity & Correlation coefficient: $-0.374, \mathrm{P}<0.01$ & Correlation coefficient: $-0.111, P>0.05$ \\
\hline 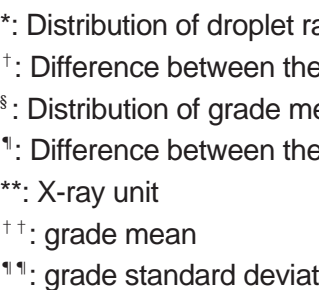 & $\begin{array}{l}\text { adiodensities with time in eithe } \\
\text { casting group and plugging } g \\
\text { ean or grade SD with time in e } \\
\text { casting group and plugging g }\end{array}$ & $\begin{array}{l}\text { casting group or plugging group } \\
\text { oup in droplet radiodensities } \\
\text { her casting group or plugging group } \\
\text { oup of either grade mean or grade SD }\end{array}$ & \\
\hline
\end{tabular}



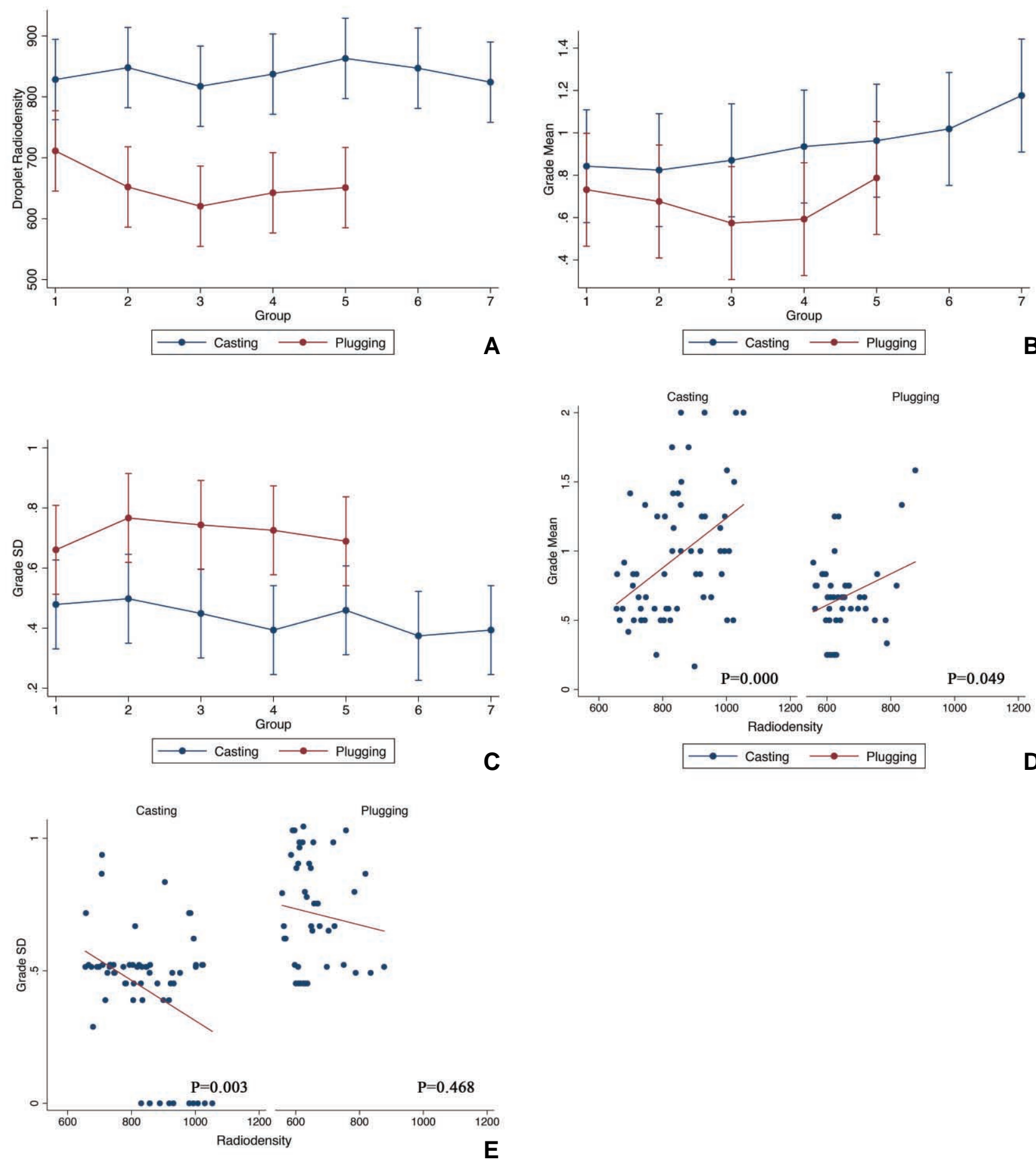

Fig. 3. (A) Sequential change of droplet radiodensity with $95 \%$ confidence intervals (Cls) in casting mode and plugging mode (repeated measures). The unit of radiodensity is X-ray unit standing for X-ray attenuation. (B) Sequential change of the mean value of the microcatheter radiographic grade with $95 \%$ confidence intervals (Cls) in casting mode and plugging mode (repeated measures). (C) Sequential change of the standard deviation (SD) of the microcatheter radiographic grades with $95 \%$ confidential intervals (Cls) in casting mode and plugging mode (repeated measures). (D) Scatter plot of the droplet radiodensity and the mean of the microcatheter radiographic grades. (E) Scatter plot of the droplet radiodensity and the standard deviations (SDs) of the microcatheter radiographic grades. 
decreased (Fig. 3E). Statistical analysis showed a significantly positive correlation between radiodensity and mean $(\mathrm{P}<0.01)$, and a significantly negative correlation between radiodensity and $\mathrm{SD}(\mathrm{P}<0.01)$ (Table 1$)$.

The same correlations were observed in the plugging mode, except that there was insignificant correlation between the radiodensity and the SD (Fig. 3D, E; Table 1).

\section{DISCUSSION}

During an early phase of introducing Onyx as a new embolic material for arteriovenous malformation embolization, many interventionists were asked to repeatedly turn the injection syringe upside down in a slow motion, especially during the injection-pause period. We thought that this action could help minimize inevitable early sedimentation problem, at least within the syringe. The necessity could be inferred by the fact that the manufacturer company recommended restirring of the well-stirred Onyx vial if injection could not be initiated within five minutes after drawing into the injecting syringe. We experienced an exaggeration of the problem when injecting through relatively small feeders requiring a low injection rate or after several pauses of injection during a plug formation.

Regarding liquid embolic materials, researchers have investigated the flow pattern of embolic agents in circulating blood [8]. However, the radiopacity issue of Onyx remains under-investigated. This in vitro experiment was designed as a fast and reproducible experiment model to enable the study of the injection characteristics of liquid embolic agents including Onyx, and thus to investigate how to improve the product and the embolization technique.

The experiment was aimed to simulate human anatomy and real procedure. In our benchtop setup, there were multiple curves especially at the head portion of the microcatheter, which became the target of radiographic imaging simulating the lateral angiographic imaging of a typical BAVM embolization. The injection of Onyx was divided into a casting mode and a plugging mode $[3,7]$.

The fixed flow rate of $0.05 \mathrm{~mL} / \mathrm{min}$ was based on research and the fact that the injection rate may vary according to the clinical and procedural situation and individual operator's experience [7]. The company's recommended injection rate was approximately 0.1 $\mathrm{mL} / \mathrm{min}$ (not exceeding $0.3 \mathrm{~mL} / \mathrm{min}$ ) and two previous studies reported a flow rate of $0.1-0.2 \mathrm{~mL} / \mathrm{min}[4,9]$. However, the usual injection rate at our institution was lower because we wanted to take advantage of the non- adherent nature of Onyx $[10,11,6]$.

For the plugging period, we tried to simulate a period when we created a plug around the tip of the injecting microcatheter [1-3]. After completion of casting mode, we waited for $1 \mathrm{~min}$ without injection followed by repeated injections for a brief period of $2 \mathrm{~s}$ while collecting a total of five Onyx droplets. Although the injection could be ceased up to $2 \mathrm{~min}$ [1], we chose to pause for $1 \mathrm{~min}$ because use of shorter plugging modes simplified the experiment. Furthermore, a shorter plugging period facilitates better identification of issues associated with the dose for Onyx visualization than a longer plugging period.

In order to analyze the behavior of the Onyx in the microcatheter lumen, we measured both the radiodensity of the collected Onyx droplets and the radiopacity of the microcatheter by taking radiographs together. Radiodensity change of the serially collected Onyx droplets was measured to plot a time-radiodensity curve allowing us to observe the change of droplet radiodensity over time and the difference of the droplet radiodensity between the two injection modes. The droplet radiodensities in the casting mode were significantly higher than those in the plugging mode. This could be explained by the increased tantalum powder present when Onyx was continuously injected.

The clinical phenomenon of poor radiopacity with Onyx, which we speculate mainly results from the various degree of sedimentation of tantalum powder in different portions of the microcatheter, requires quantification. No previous method has been published to measure the radiodensity fluctuation pattern. We designed an easy-to-adapt grading system to evaluate the visualization of the microcatheter. The distal segment of the microcatheter was divided into 12 segments according to its shape and length and the radiodensity of each segment was graded into three levels. The total visualization and the evenness of the microcatheter were respectively evaluated by the mean of all 12 grades and its SD.

In the casting mode, during injection of Onyx over time, the microcatheter had a gradual higher mean and lower SD. This meant that the visualization of the catheter became better as the injection continued due to forced advancement of the sedimented tantalum powder and the introduction of well-mixed fresh Onyx into the portion. On the contrary, the visualization of the microcatheter in the plugging mode over time was less satisfactory. Repeated measures of SD showed a significant reduction of SD with time in the plugging mode. This may be due to tantalum sedimentation due to 
stagnation of the Onyx in the portion. Discontinuous 2-s jerk injections were not able to improve the visualization.

According to our experience and the current evidence, the precipitation of tantalum in the mixture of Onyx led to the change of the microcatheter contents and its visualization. Brisk jerk injection was not able to improve the visualization of the catheter during the plugging mode. In this setting, the "two-catheter technique" might help reduce the waiting time and improve the visualization [13].

From our observation, we could hypothesize that the sedimentation could be minimized using an increased flow rate of 2-s injection for the plugging mode. We attempted a very short but rather jerky injection during the creation of a plug and could increase the radiopacity of the tiny Onyx cast. However, this should be confirmed with further experimentation.

As speculated from clinical observations, we confirmed that the inhomogeneous radiopacity of the injecting Onyx material for the embolization of BAVM was due to early precipitation of the tantalum powder within the microcatheter lumen. In addition, we found additional interesting behavior of the Onyx mixture. First, the radiopacity of the injecting Onyx seemed to be higher and more homogeneous during continuous injection. However, it dropped significantly right after stopping the injection and its lower radiopacity was maintained during plugging period. Second, the lower radiopacity of the injecting Onyx seemed to recover when the continuous injection re-initiated. However, there were fluctuations of radiopacity due to forced advancement of the Onyx with sedimented tantalum powder in the dependent part of the multiple curves of the microcatheter.

Our experiment has some limitations: (1) the current in vitro design did not factor in a blood flow factor, which could significantly influence the behavior of injecting Onyx; (2) there could be a limitation in the characterization of other physical feature of the injecting Onyx as we could only measure the radiodensity of the collected Onyx droplet. Change in the portion of tantalum powder might influence on the injection friction as well; (3) as a surrogate indicator of tantalum powder volume, we tried to measure the individual droplet weight; however, we could not get a reliable result; (4) the flow rate of $0.05 \mathrm{~mL} / \mathrm{min}$ might be not a representative injection rate; and (5) our test setting could be too simplified and should be repeated with different injection settings.

In conclusion, because of early precipitation of tantalum powder, the radiopacity of Onyx can vary significantly over time. Radiopacity decreases more prominently during plugging modes, which is characterized by pauses between injections. The problem could be addressed by improving tantalum powder sedimentation and the injection technique to minimize early sedimentation of the powder, especially during the plug formation period.

\section{References}

1. Katsaridis V, Papagiannaki C, Aimar E. Curative embolization of cerebral arteriovenous malformations (AVMs) with Onyx in 101 patients. Neuroradiology 2008;50:589-597

2. Mounayer C, Hammami N, Piotin M, Spelle L, Benndorf G, Kessler I, et al. Nidal embolization of brain arteriovenous malformations using Onyx in 94 patients. AJNR Am J Neuroradiol 2007; 28:518-523

3. van Rooij WJ, Jacobs S, Sluzewski M, van der Pol B, Beute GN, Sprengers ME. Curative embolization of brain arteriovenous malformations with onyx: patient selection, embolization technique, and results. AJNR Am J Neuroradiol 2012;33:12991304

4. Terada T, Nakamura Y, Nakai K, Tsuura M, Nishiguchi T, Hayashi S, et al. Embolization of arteriovenous malformations with peripheral aneurysms using ethylene vinyl alcohol copolymer. Report of three cases. J Neurosurg 1991;75:655-660

5. Yamashita K, Taki W, Iwata H, Nakahara I, Nishi S, Sadato A, et al. Characteristics of ethylene vinyl alcohol copolymer (EVAL) mixtures. AJNR Am J Neuroradiol 1994;15:1103-1105

6. Siekmann R. Basics and Principles in the Application of Onyx LD Liquid Embolic System in the Endovascular Treatment of Cerebral Arteriovenous Malformations. Interv Neuroradiol 2005; 11:131-140

7. van Rooij WJ, Sluzewski M, Beute GN. Brain AVM embolization with Onyx. AJNR Am J Neuroradiol 2007;28:172-177

8. Lv X, Wu Z, Li Y. Arteriovenous malformation in the brain: a theoretical study explaining the behavior of liquid embolic agents during endovascular treatment. Neuroradiol J 2013;26:661-668

9. Weber W, Kis B, Siekmann R, Kuehne D. Endovascular treatment of intracranial arteriovenous malformations with onyx: technical aspects. AJNR Am J Neuroradiol 2007;28:371-377

10. Chaloupka JC, Huddle DC, Alderman J, Fink S, Hammond R, Vinters HV. A reexamination of the angiotoxicity of superselective injection of DMSO in the swine rete embolization model. AJNR Am J Neuroradiol 1999;20:401-410

11. Murayama Y, Vinuela F, Ulhoa A, Akiba Y, Duckwiler GR, Gobin YP, et al. Nonadhesive liquid embolic agent for cerebral arteriovenous malformations: preliminary histopathological studies in swine rete mirabile. Neurosurgery 1998;43:1164-1175

12. van Beijnum J, van der Worp HB, Buis DR, Al-Shahi Salman R, Kappelle LJ, Rinkel GJ, et al. Treatment of brain arteriovenous malformations: a systematic review and meta-analysis. JAMA 2011;306:2011-2019

13. Durst CR, Starke RM, Gaughen J, Evans AJ. A method for complete angiographic obliteration of a brain arteriovenous malformation in a single session through a single pedicle. $J$ Clin Neurosci 2015;22:391-395 